\title{
Georges Dumézil e la psicologia storica: uno scambio epistolare con Ignace Meyerson
}

Stefano Acerbo

\section{(2) OpenEdition}

\section{Edizione digitale}

URL: http://journals.openedition.org/mythos/521

DOI: $10.4000 /$ mythos. 521

ISSN: 2037-7746

\section{Editore}

Salvatore Sciascia Editore

\section{Edizione cartacea}

Data di pubblicazione: 1 décembre 2016

Paginazione: $215-228$

ISBN: 978-88-8241-476-4

ISSN: 1972-2516

\section{Notizia bibliografica digitale}

Stefano Acerbo, « Georges Dumézil e la psicologia storica: uno scambio epistolare con Ignace Meyerson », Mythos [Online], 10 | 2016, online dal 24 septembre 2019, consultato il 28 septembre 2019. URL : http://journals.openedition.org/mythos/521 ; DOI : 10.4000/mythos.521 


\section{Georges Dumézil e la psicologia storica: uno scambio epistolare con Ignace Meyerson ${ }^{1}$}

\section{Stefano Acerbo}

\begin{abstract}
Riassunto
A partire dall'esame di tre lettere inviate da Georges Dumézil a Ignace Meyerson, questo articolo si propone di indagare il tentativo dell'indoeuropeista di riconsiderare i fondamenti metodologici alla base della sua teoria trifunzionalista. Aprendosi a un dialogo con differenti approcci intellettuali, come la psicologia storica ma anche il marxismo, Dumézil cerca di superare i limiti teorici imposti da un approccio puramente durkheimiano ai risultati empirici cui erano giunte le sue ricerche. Questa revisione metodologica ebbe un risultato solo in parte positivo. Tutte le trascrizioni di questi documenti inediti sono pubblicate integralmente in appendice.
\end{abstract}

\begin{abstract}
Taking into account three letters sent by Georges Dumézil to Ignace Meyerson, this study aims to investigate the methodological reassessments made by the indoeuropeist of the trifunctionalist theory. Engaging in a dialogue with different intellectual approaches, like historical psychology but also marxism, Dumézil tries to overpass the strict limits imposed by a durkheimian approach to the empirical results of his studies. The reassessment was only in part successful. The transcriptions of all these unpublished documents are published in appendix.
\end{abstract}

\section{Parole chiave}

Storia della cultura - Trifunzionalismo - Psicologia storica - Sociologia - Marxismo

\section{Keywords \\ History of culture - Trifuncionalism - Historical psychology • Sociology - Marxism}

I

percorso intellettuale che ha condotto Georges Dumézil a concepire e, poi, a rivedere a più riprese la teoria "trifunzionale" è stato oggetto di vari studi a partire da prospettive diverse. Si tratta, infatti, di una questione di grande interesse storico-culturale in quanto permette di cogliere le fondamenta teoriche, i presupposti ideologici e i criteri di metodo all'origine di una corrente di studi che ha avuto una notevole diffusione.

La lettura critica di alcuni documenti inediti scritti da Dumézil può offrire un contributo forse limitato, ma di importanza non trascurabile, per indagare un fondamentale passaggio all'interno della produzione dell'indoeuropeista, che si registra tra la fine degli anni '40 e l'inizio degli anni '50. All'interno dell'abbondante corrispondenza conservata nel Fonds Ignace Meyerson, vi sono tredici lettere inviate allo psicologo proprio da Georges Dumézil². La lettura

1 Questo articolo nasce da un lavoro di studio e catalogazione di alcuni documenti presenti nel Fonds Ignace Meyerson conservati presso la sede di Pierrefitte delle Archives Nationales, condotto durante un periodo di Erasmus Placement presso il Centre ANHIMA (UMR 8210). Ringrazio per l'opportunità offertami i professori Cléo Carastro, Riccardo Di Donato e Andrea Taddei. Desidero ringraziare anche les Archives Nationales per avermi concesso di pubblicare la trascrizione dei documenti (Rèference: AN/DIRP/DE-VB/100-850-930)..

2521 AP. Meyerson (Ignace) 19920046/51. A proposito delle Archives Ignace Meyerson si vedano Di Donato 1990, Di Donato 2013, 175-185. 
critica di questi documenti mostra come la psicologia storica abbia, probabilmente, avuto un ruolo non secondario nel tentativo operato dall'indoeuropeista di trovare un difficile equilibrio tra un fondamento teorico di natura sociologica, e quindi storica, e un'analisi di tipo funzionale, che, per certi versi, anticipa le teorie strutturaliste; tentativo che si tradurrà in un orientamento di indagine parzialmente rinnovato.

Prenderò in considerazione tre lettere inviate da Dumézil a Meyerson: collocate in un lasso di tempo piuttosto ristretto, dal febbraio 1949 al novembre 1950, che permettono di cogliere lo sforzo intellettuale prodotto dall'indoeuropeista al fine di superare un'aporia di natura teorica e metodologica di cui aveva preso coscienza. Lo studio dello sviluppo storico delle funzioni psicologiche poteva affrancare l'indoeuropeista da un'impostazione strettamente sociologica, i cui fondamenti teorici contrastavano con i risultati empirici cui Dumézil era giunto. Pertanto, nella psicologia storica Dumézil sembra intuire un possibile contrappunto utile a rendere più solido il proprio metodo di indagine, senza, peraltro, escludere l'apporto di correnti di pensiero del tutto estranee al proprio profilo intellettuale.

Di questo avvicinamento agli studi di Meyerson così come della profonda problematizzazione delle categorie su cui è costruita la teoria del trifunzionalismo, non resta molto negli scritti pubblicati. Perciò la chiarezza e la franchezza con cui in queste lettere Dumézil pone questioni cruciali risultano un'importante testimonianza per giudicare in che misura lo studioso sia riuscito effettivamente a venire a capo dell'aporia da lui stesso indicata. Il passaggio che si registra nella produzione intellettuale dell'indoeuropeista all'inizio degli anni ' 50 , e che è stato individuato da chi ha ricostruito il suo profilo intellettuale ${ }^{3}$, pur traendo origine proprio dalla presa di coscienza di questi nodi problematici, come vedremo, non porta a un loro effettivo superamento, almeno nei termini che lo studioso poneva in queste lettere.

Il piccolo corpus epistolare tra George Dumézil e Ignace Meyerson si apre con sei lettere che testimoniano una corrispondenza piuttosto intensa tra il febbraio del 1949 e il novembre 1950; in particolare tre di esse mostrano un effettivo contatto intellettuale tra i due studiosi e si prestano a una proficua indagine ${ }^{4}$. Si tratta della prima lettera datata al 6/02/1949, della quarta risalente al 29/04/1949 e della sesta del 18/11/1950. Tutte e tre sono scritte sul recto e sul verso di fogli grandi da lettera ${ }^{5}$, la cui superficie è stata utilizzata da Dumézil quasi per intero se si eccettua un certo margine vuoto solamente nella parte sinistra del foglio. La scrittura è generalmente rapida e la grafia non è particolarmente curata, al punto da creare, in alcuni casi, serie difficoltà di lettura. Si registrano casi di cancellatura e di correzioni o aggiunte intralineari o marginali in misura, però, limitata, il che fa pensare a una stesura piuttosto sicura senza molti ripensamenti ${ }^{6}$.

3 García 2001, 37-38.

4 Il motivo all'origine dello scambio epistolare verte intorno alla nomina di Lucien Gerschel al CNRS. Questa questione di politica accademica, non rilevante ai fini di questo studio, occupa interamente le tre lettere qui non riportate oltre che parti di quelle che saranno oggetto di esame.

5 Si tratta di fogli A4.

6 Il testo integrale delle tre lettere è fornito in appendice al termine dell'articolo. 


\section{Un nodo aporetico}

$\mathrm{L}^{\prime}$ occasione dell'incontro è fornita da una recensione al libro di Dumézil intitolato Loki', firmata da Meyerson nel Journal de Psychologie Normale et Pathologique ${ }^{8}$. Questo libro, che analizza i paralleli tra le leggende del dio scandinavo Loki e il personaggio dei racconti caucasici dei Narti Syrdon, mostra le difficoltà e l'impasse cui era giunto l'indoeuropeista nei suoi studi, tanto che si chiude con una vera e propria aporia':

Finalement, nous n'avons aucun moyen d'expliquer entièrement le parallélisme Loki-Syrdon. Nous restons avec des constatations négatives : les deux dossiers ne sont pas séparables, mais ne paraissent pas dériver d'un prototype commun, ni non plus dériver l'un de l'autre; et pourtant des simples considérations de structure sociale ne suffisent pas à rendre compte de l'aspect fraternel qu'ils présentent (p. 266).

Lo studioso sembra mostrare una certa insoddisfazione per le spiegazioni puramente sociologiche come base per giustificare il ripresentarsi dell'ideologia trifunzionale nei popoli parlanti lingue indoeuropee. Alla luce della conclusione di Loki non apparirà pertanto banale quanto scritto all'inizio della prima lettera datata 6 febbraio 1949 (Appendice: testo numero 1): dopo aver ringraziato Meyerson per la generosa recensione al suo libro, Dumézil palesa il proprio rammarico ${ }^{10}$ :

Si j'étais plus jeune, j'essaierais de doubler d'analyses psychologiques l'exégèse un peu lourdement sociale que je fais des mythes. Mais l'âge est l'âge : je puis encore fonctionner dans le sens où je suis " remonté ", mais impossible de modifier le mécanisme.

Fino a questo livello cronologico Dumézil aveva considerato l'ideologia trifunzionale, che egli pensava di poter rintracciare presso gran parte dei popoli indoeuropei, un riflesso di reali condizioni sociali, ipotizzando, quindi, una società indoeuropea che si basava su un'effettiva divisione in tre classi della società ${ }^{11}$.

Proprio nello stesso anno, il 1949, Dumézil aveva pubblicato L'heritage indo-européen à $R_{o m e}{ }^{12}$, una sorta di introduzione retrospettiva ai suoi libri precedenti dove aveva ancora sostenuto questa posizione. Ma se Dumézil si poteva dire compiaciuto dei risultati empirici cui riteneva di essere giunto nelle sue ricerche, non mostrava, però, una pari soddisfazione per il metodo seguito come emerge anche dal passo della lettera del 6 febbraio " 49 preso in considerazione in precedenza (je puis encore fonctionner dans le sens où je suis "remonté", mais impossible de modifier le mécanisme). Tale insoddisfazione nasce dalla presa di coscienza di una serie di nodi problematici, di natura teorica, con cui si scontravano i suoi studi.

Dumézil considerava l'ideologia trifunzionale non una struttura universalmente operante nella mente umana, ma un'eredità storica propria dei soli popoli parlanti lingue del ceppo in-

7 DuMÉZIL 1948. Riedizione nel 1986.

8 Meyerson 1949, 122-123.

9 Si veda a proposito RIVIÈRE 1979, 95-96.

10 Per la traslitterazione delle lettere qualora non mi sia stato possibile leggere una parola mi sono servito delle due parentesi quadre con i tre punti di sospensione [...].

11 García Quintela 2001, 37-38.

12 DuMÉZIL 1949. 
doeuropeo, eredità proveniente da un'antica fase preistorica comune ${ }^{13}$. La spiegazione ultima dell'esistenza di tale ideologia fu, anche in seguito, sebbene con oscillazioni varie, di natura sociologica: gli indoeuropei in origine sarebbero stati divisi in tre classi ${ }^{14}$.

Se la struttura sociale è il fondamento dell'ideologia trifunzionale risulta assai difficile spiegare le ragioni per le quali tale ideologia si ripresenta anche laddove la struttura sociale non è per nulla tripartita, come nella Roma repubblicana. L'ipotesi che l'ideologia, e non delle semplici survivances, possa trasmettersi indipendentemente dalle strutture sociali è contraria alla sociologia di matrice durkheimiana su cui Dumézil ancora fondava i suoi studi ${ }^{15}$. Proprio per tale ragione, a questa altezza cronologica, lo studioso aveva cercato di uscire da una prospettiva strettamente sociologica.

\section{Dumézil e l'incontro con la psicologia storica}

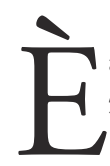

alla luce di questo tentativo che si può pienamente comprendere l'interesse e l'apprezzamento mostrato da Dumézil per gli articoli di Meyerson nella lettera datata 24 aprile 1949 (Appendice: testo numero 2).

Il giudizio dell'indoeuropeista è sincero, sebbene, allo stesso tempo, non rifletta una valutazione lungamente ponderata degli studi condotti da Meyerson ${ }^{16}$. Dumézil, infatti, nell'esprimere tale giudizio, coglie solo una parte del portato delle tesi di Meyerson, soffermandosi esclusivamente su quanto poteva essere funzionale a offrire un supporto teorico alle proprie ricerche. Dumézil, in base alla propria esperienza empirica, concorda sul fatto che l'evoluzione storica di quelle che Meyerson chiama funzioni psicologiche, termine mai impiegato dall'indoeuropeista, non corrisponda a un continuo progresso ma comporti discontinuità e crisi. Di tali discontinuità la lettera offre una descrizione piuttosto vaga.

Quant aux discontinuités, vous me trouvez conquis d'avance par mes expériences personnelles de chercheur et par les quelques coups d'œil que j'ai pu jeter sur le monde ; votre refus du " rationalisme simpliste " et de l'" empirisme naïf » qui intéressent tant de discussions est libératoire, cette mise en évidence des rythmes, des crises, des risques, des métamorphoses, des mutations ; à coté des successions, des progrès, des développements évolutifs, rétablit l'équilibre systémique et philosophique de la recherche.

13 García Quintela 2001, 39.

14 Il campo di prova di tale teoria è offerto dall'annalistica romana più antica. Dumézil, sebbene nel tempo abbia sfumato l'idea di un'originaria società romana divisa in tre classi, ancora nella sua intervista con Éribon si esprime nei seguenti termini: Era forte la tentazione di arrivare alla conclusione, secondo la quale prima della storia, alla nascita di Roma - e senza dubbio in ogni società latina di cui Roma è solo il caso noto - la popolazione era inizialmente divisa in tre classi come gli indiani o i celti e che l'annalistica, o ciò che la precedette, retta dalla preoccupazione di radicare le tradizioni preromane nel contesto e nel territorio italiano, aveva protetto la divisione trifunzionale con la divisione etnica. Fu solo una tentazione. Il mio sentimento è cambiato e si è attenuato nel corso dei decenni. Ma a conti fatti penso non sia scandaloso cedere a quella tentazione. [...] Ma come capita di frequente, l'ideologia è vissuta più a lungo nell'immaginario che nella realtà e, benché sia inutile, la leggenda della fondazione di Roma da parte dei protetti di Giove, degli specialisti della guerra e di una piccola parte ricca di armenti, è sopravvissuta - come è rimasto il ricordo del fatto che le tre tribù primitive presto dimenticate avevano in un modo o nell'altro attribuiti funzionali - mantenendo le tre funzioni. DumÉzIL 1992, 88-89.

15 García Quintela 2001, 34-36.

16 Meyerson 1948. 
In tali discontinuità Dumézil vede il rifiuto del "razionalismo semplicista" e dell'“empirismo naif" propri di molta parte della discussione scientifica. Il fatto che lo studioso possa giungere a definire "liberatoria" questa messa in evidenza delle crisi, dei ritorni all'indietro, a fianco dei successi e dei progressi scientifici, deriva da una lettura dell'opera di Meyerson che dipende solo parzialmente dal proprio conservatorismo, ma che mi sembra, piuttosto, mostrare un tentativo di trovare nelle più recenti teorie una giustificazione non solamente empirica ai propri studi.

La presenza di discontinuità, di possibili ritorni all'indietro, poteva fornire un argomento a sostegno dell'individuazione del riaffiorare di un'ideologia trifunzionale di derivazione protostorica anche in contesti assai mutati.

Certamente si trattava di una semplice suggestione e come tale non bastava a costituire una base scientifica di cui lo studioso poteva avvalersi quale supporto alle proprie tesi.

In queste prime due lettere datate al 1949, Dumézil, sebbene si renda conto della necessità, per salvare le sue teorie, di superare l'eccessivo schematismo sociologico, non mostra ancora una vera messa in discussione dei presupposti su cui fondavano i propri studi. Si è già visto come nel contemporaneo L'heritage indo-européen lo studioso considerasse ancora l'ideologia trifunzionale un semplice riflesso di strutture di natura sociale. E nella lezione inaugurale al Collège de France, datata al dicembre del $1949^{17}$, aveva superato l'impasse cui era giunto al termine dell'opera Loki nel modo più semplice, ovvero accettando quanto rifiutato in precedenza: la presenza di "schemi narrativi comuni" presso due popoli indoeuropei non in relazione reciproca, mostrerebbe l'esistenza di una letteratura indoeuropea, ovvero di schemi narrativi che risalgono alla civiltà indoeuropea ${ }^{18}$ e che da essa si sarebbero trasmessi ai suoi eredi storici ${ }^{19}$.

In realtà le contraddizioni e le difficoltà non solo non erano superate, ma, per il momento, neppure accantonate. Nella lettera del 18 novembre 1950 (Appendice: testo numero 3), di gran lunga la più interessante, Dumézil afferma di trovarsi, da più di un anno, in uno stato di sterilità intellettuale tale da non aver potuto preparare neppure uno scritto di base per il corso che dovrà tenere. E proprio in questo stato aporetico della riflessione dello studioso si registra il più forte punto di avvicinamento con le ricerche condotte da Ignace Meyerson. Dumézil scrive di aver comunicato il proprio interesse per l'apporto che può derivare dal tipo di psicologia praticato da Meyerson a $S$. Wikander ${ }^{20}$, il quale si era mostrato a sua volta interessato agli studi condotti dallo psicologo. Ma questo interesse intellettuale doveva essere in una certa misura condiviso: infatti Dumézil, nel prosieguo della lettera, presenta un breve schema di uno studio appena completato, ipotizzando che possa risultare interessante per Meyerson. E, in effetti, lo psicologo dovette mostrare un effettivo apprezzamento, in quanto tale articolo si trova già pubblicato nel numero di quello stesso anno del Journal de psychologie normale et pathologique da lui diretto ${ }^{21}$.

La descrizione di questo breve scritto mette chiaramente in luce quale fosse la problematica

17 DuMÉzIL 1950.

18 Si rimanda a Di Donato 1999, 56-59; 81-100 per un'approfondita disamina del dibattito intorno all'esistenza storica di una popolazione indoeuropea avente una propria cultura. La questione è stata recentemente affrontata, con il supporto di nuovi dati archeologici, da Demoule 2014.

19 Rivière 1979, 95-96.

20 Per una ricostruzione del profilo biografico e intellettuale di Stig Wikander, attivo a Uppsala e stretto collaboratore di Dumézil si rimanda a Duchesne-Guillemin 2009 e Timuş 2004.

21 DuMÉZIL $1950 \mathrm{~b}$. 
che tormentava Dumézil e a quali metodi esegetici, che possono apparire persino sorprendenti, fosse disposto a rivolgersi pur di trovare un'uscita dall'impasse cui era giunto.

Quando comparirà in rivista, l'articolo cui lo psicologo fa qui riferimento avrà il titolo Les archanges de Zoroastre et les rois romains de Cicéron. Nella breve descrizione fornita all'interno della lettera, lo studioso sostiene che la storia di Roma narrata nella Repubblica di Cicerone e la mitologia indoiranica, dopo la riforma zoroastriana, palesano una struttura comune soggetta a modificazioni parallele dettate, in un caso, da una filosofia politica, dall'altro da una mistica moralizzante. In questo contributo, e in particolare nel modo in cui esso viene presentato a Meyerson in questa lettera, l'indagine condotta da Dumézil sembra già focalizzarsi sull'evoluzione e le trasformazioni subite dall'ideologia trifunzionale all'interno dei contesti specifici dei singoli popoli parlanti lingue indoeuropee. Qui lo studioso coglie le specificità di ognuno di esso, con una conseguente rinuncia, seppure parziale, a una ricostruzione che riconduca tutte queste specificità a un unico quadro ideologico da collocarsi nella fase proto-storica comune della civiltà indoeuropea. E proprio in questo mutamento nella prospettiva di indagine si può ipotizzare un influsso esercitato dalla lettura degli scritti di Meyerson, in quanto la psicologia storica, opponendosi a ogni fissismo e considerando le funzioni psicologiche solo nel loro divenire storico, offriva un forte stimolo a considerare le forme di pensiero in stretta connessione con il contesto storico in cui esse si concretizzavano nelle opere.

Il passaggio che in questa lettera è possibile vedere in fieri giunge a compimento nel libro del 1952 Les dieux des indoeuropéens ${ }^{22}$ e consiste nell'attribuire alla sfera ideologica un'indipendenza rispetto alle condizioni sociali, venendo a trattare l'ideologia trifunzionale come un dato fattuale operante nell'evoluzione storica dei popoli indoeuropei ${ }^{23}$. Qualora si considerino solamente le opere pubblicate dallo studioso, tale passaggio risulta essere la conclusione positiva a cui giunge la problematizzazione dei presupposti su cui si basava la propria precedente opera, condotta da Dumézil alla fine degli anni ' $40^{24}$. In realtà, questo nuovo indirizzo di ricerca non era realmente risolutivo giacché l'indipendenza della sfera ideologica e, quindi, il permanere dell'ideologia trifunzionale nei popoli indoeuropei, senza un legame con la reale struttura sociale, necessitava di una spiegazione teorica che Dumézil non trovò mai realmente, come viene riconosciuto nelle Entretiens con Didier Éribon, sorta di riconsiderazione retrospettiva della propria opera al termine della vita ${ }^{25}$.

22 DumÉZil 1952b

23 García Quintela 2001, 37-38.

24 In tale maniera viene, infatti, presentata da García Quintela 2001, 36-39.

25 La mancanza di una spiegazione a questo nodo problematico viene palesata nella risposta di Georges Dumézil alla domanda di Didier Éribon. (D.É.): Resta un problema non secondario: in che modo questa ideologia e le sue realizzazioni, specialmente quelle letterarie, sono sopravvissute in quasi tutti gli eredi degli "indoeuropei comuni", pur adattandosi a luoghi e tempi nuovi?. (G.D): In effetti si tratta di un immenso problema che i "fatti comparativi", aggiornati dal mio lavoro e da quello di altri ricercatori, pongono, ma che non siamo in grado di risolvere. Può darsi che i successori di Changeux scoprano quali siano i meccanismi cerebrali che permettono queste conservazioni e questi adattamenti. DumÉzIL 1992107. 


\section{Una possibile spiegazione marxista}

I

1 testo inedito della lettera inviata da Meyerson il 18 novembre 1950 si rivela un documento di straordinaria importanza per una comprensione più profonda di questa fase cruciale all'interno del percorso intellettuale dell'indoeuropeista, in quanto mostra come, a questa altezza cronologica, Dumézil, oltre a riconoscere l'esistenza di questo nodo problematico, fosse alla ricerca di una spiegazione che permettesse di superarlo.

Proprio nel contatto con Meyerson lo studioso cercava un possibile supporto che gli permettesse di superare questa difficoltà, dimostrandosi persino interessato a ricevere suggestioni che provenivano da una tradizione a lui del tutto estranea, quale era il pensiero marxista.

En attendant J'ai achevé un petit papier qui vous intéressera peut-être (je pense qu'il va dans le sens de votre revue) où j'essaie de montrer comment, représentant l'histoire royale de Rome (dans De Republica) en fonction de préoccupations philosophiques, Cicéron (qui fait subir des retouches exactement aux mêmes endroits (et dans le même sens) où la réforme zoroastrienne, dans ses préoccupations morales, a retouché la mythologie $^{26}$ fonctionnelle des Indo-Iraniens. Comme je pense que cette mythologie - (où théologie) indo - iranienne et cette histoire royale romaine sont bâties sur la même structure de pensée, le parallélisme des corrections qu'elles subissent l'une et l'autre, sont l'effet ici d'une philosophie politique, là d'une mystique moralisante, est un phénomène curieux, que je voudrais dégager, laissant aux autres le soin d'y réfléchir. Je serais curieux de voir l'explication marxiste de ces adaptations et rajeunissements de "superstructures" qui, ici et là, ne correspondent plus, depuis longtemps, à l'actualité sociale.

Tale interessamento, così sorprendente, sembra andare nella direzione di un'intuizione avuta da Momigliano in un articolo del $1983^{27}$ in cui si traccia un profilo intellettuale dell'indoeuropeista francese. Lo storico italiano, facendo riferimento al saggio Le Troisième Souverain ${ }^{28}$ del 1949 , in cui veniva riconosciuto un elemento di sovranità anche alla classe dei produttori, si era chiesto se alla fase "gerarco-fascista", che Momigliano riteneva di aver identificato nella produzione di Dumézil prima della guerra, non avesse fatto seguito una fase vagamente marxista. È solo un'ipotesi posta nella forma di una domanda aperta, ma Momigliano sembra prendere in considerazione l'idea che sollecitazioni esteriori, da individuare nelle teorie marxiste, avessero potuto contribuire ad affinare l'analisi interna degli elementi di sovranità, che rappresenta uno dei contributi più importanti portati dallo studioso francese ${ }^{29}$.

L'interesse che emerge da questa lettera, piuttosto che indicare improbabili avvicinamenti politici ${ }^{30}$ mostra come in questa fase della sua vita Dumézil si dimostrasse aperto ad accogliere

26 È cancellata la parola <théologie>.

27 Momigliano 1983.

28 DuMÉZIL $1949 \mathrm{~b}$.

29 Momigliano 1983, 337: Almeno nel saggio del 1949 sul Troisième Souverain Dumézil sembra ora disposto ad ammettere che un elemento sovrano sia fornito anche dalla classe dei produttori, la terza classe. Non è del tutto fuori posto domandarsi se alla fase gerarchico-fascista della interpretazione della struttura sociale indo-europea non succeda una fase vagamente marxista in cui i produttori hanno la loro voce nella sovranità. Ma quali che siano le sollecitazioni esteriori, non è per me dubbio che l'analisi interna degli aspetti della sovranità rappresenta uno dei contributi più importanti di Dumézil.

30 Dumézil non ebbe mai simpatie marxiste o progressiste, ma ebbe a lungo una visione fortemente conservatrice. 
contributi intellettuali provenienti da aree di pensiero differenti. Nel 1950 lo studioso si era già allontanato, e da molti anni, da un impegno attivo nella politica e intratteneva relazioni intellettuali anche con studiosi gauchisti.

La domanda che Dumézil rivolge a Meyerson va pertanto considerata seriamente. Dumézil sapeva che Meyerson, iscritto al PCF, era in stretto contatto con pensatori aventi anche riferimenti marxisti e, in alcuni casi, a loro volta gravitanti intorno al PCF e, pertanto, poteva sperare realmente di ricevere una risposta da parte di Meyerson e trovare, all'interno di una spiegazione di origine marxista, un appoggio alla propria teoria trifunzionalista.

La curiosità da parte di Dumézil per i possibili apporti che le teorie marxiste potevano offrire ai suoi studi, mostrata in questa lettera, rende meno azzardata la domanda che Momigliano poneva, offrendo una testimonianza concreta di quanto lo storico italiano aveva intuito ${ }^{31}$.

\section{Un nuovo orientamento di ricerca: Les dieux des Indo-européens}

prescindere da possibili e inattese aperture intellettuali, l'interrogativo espresso da
Dumézil in termini marxisti rappresenta il nodo problematico che sta alla base dell'in-
teresse di questo studioso per la psicologia storica praticata da Meyerson.

La psicologia storica poteva offrire un sostegno al tentativo di Dumézil di smarcarsi da spiegazioni eccessivamente sociologiche, e pertanto ci si può chiedere in quale misura l'incontro con Ignace Meyerson abbia contribuito alla svolta che si registra nei primi anni ' 50 nell'attività di Dumézil. Per rispondere a questo interrogativo si può prendere a saggio il volume del 1952 Les dieux des Indo-européens che è stata individuata come il punto che segna definitivamente il cambiamento di prospettiva nell'opera dello studioso ${ }^{32}$.

A dispetto del titolo, quest'opera evita ricostruzioni miranti a risalire a rappresentazioni attribuibili a una fase protostorica. I primi tre capitoli proseguono nel solco già indicato all'interno dell'articolo apparso nel 1950 proprio sul Journal de Psychologie e di cui si parla nella lettera a Meyerson ${ }^{33}$. La comparazione, sostanzialmente operata tra il pantheon romano, quello indoiranico e quello scandinavo, mostra non solo il ripresentarsi di una struttura trifunzionale ma anche il suo mutarsi in relazione all'evoluzione storica delle diverse branche degli Indoeuropei ${ }^{34}$. La filiazione genealogica, che deriva in ultima istanza dalla linguistica, resta comunque il fondamento delle teorie di Dumézil. Il fatto che tanto presso i Romani, quanto in Scandinavia, e presso gli indoiranici, si possano ritrovare tre livelli teologici, considerati da Dumézil non ricostruzioni artificiali ma dati immediati, viene giustificato con la presenza d'une seule et même structure, héritée et maintenue par les uns et par les autres à partir de leur commune préhistoire (p. 36).

L'origine della struttura trifunzionale è sempre fissata nel passato preistorico indoeuropeo, e il problema di come questa struttura ideologica possa essersi trasmessa e mantenuta a distanza

Non è qui il caso di riprendere la tormentata questione sulle ambigue scelte politiche operate da Dumézil. Si rimanda a Ginzburg 1984, Momigliano 1983, Ampolo, Di Donato 1985, Éribon 1992.

31 Contra si veda ÉribOn 1992, 46-47.

32 García Quintela 2001, 37.

33 DumÉzIL 1950b.

34 Il y aura d'utiles confrontations à faire, dans le tableau que nous venons de dessiner, et en tenant compte de l'évolution historique des diverses branches des Indo-Européens. DuméZIL 1952, 74. 
di secoli o millenni, problema che Dumézil palesava con chiarezza nelle sue lettere a Meyerson non viene realmente risolto. È tale mancanza di spiegazione a portare l'indoeuropeista, nel terzo capitolo, quello maggiormente teorico, a prendere le distanze, in maniera assai rispettosa, dal metodo storico. A tal fine lo studioso contrappone a questo metodo un approccio che anticipa, per certi versi, le teorie strutturaliste poiché individua nella struttura in quanto tale l'oggetto principale di una ricerca scientifica, mentre i problemi dell'origine precisa degli altri elementi possono restare privi di soluzione senza per questo compromettere lo studio principale ${ }^{35}$. Tale possibile approccio viene, comunque, considerato in maniera critica e l'indoeuropeista non mostra una totale adesione a esso, ma rappresenta solo un contrappunto al precedente metodo storico.

Una spiegazione "strutturalista", infatti, implica l'esistenza di strutture mentali, che, in una prospettiva non razzista o genetica, dovrebbero essere universali. Dumézil ha sempre rifiutato questa possibilità, considerando il trifunzionalismo fondamento comune solo ai popoli indoeuropei ${ }^{36}$. Anche per questo motivo lo studioso ha sempre preferito parlare di ideologia indoeuropea piuttosto che di mentalità indoeuropea ${ }^{37}$. Il problema, non solo e non tanto dell'origine, quanto della natura di questa ideologia e del suo perdurare e ripresentarsi non viene però risolto e rimane un dato accettato come oggettivo e che non necessita di spiegazione, in quanto supportato da un numero elevato di parallelismi tra civiltà differenti ma aventi lingue dello stesso ceppo.

Mi sembra che il contributo più innovativo contenuto nel libro Les dieux des Indo-européens si possa trovare nel quarto capitolo. In questo vengono trattate le caratteristiche delle divinità romane, le cui specificità erano tali da poter mettere in crisi l'ipotesi di una derivazione indoeuropea. In particolare le teorie primitiviste, in un'ottica evoluzionistica, tentavano di riconnettere gli attributi di queste divinità a una fase molto antica dello sviluppo delle funzioni religiose, venendo, pertanto, a escludere la possibilità di una loro origine indoeuropea. Per contrastare queste teorie e riaffermare il carattere indeuropeo del pantheon romano, Dumézil non ricerca esempi di trifunzionalismo, ma tenta di mostrare come le specificità delle divinità romane non derivino da un loro primitivismo ma siano dovute alle caratteristiche specifiche della cultura romana. In realtà nella maggior parte degli esempi, bisognerebbe semplicemente parlare di società romana perché Dumézil offre per lo più spiegazioni propriamente sociali. Ma nell'indicare questi rapporti di servizio e subordinazione propri di certe divinità funzionali e che hanno un chiaro referente nella società romana, lo studioso individua anche una mentalità prudens, che si riflette nella società reale così come nei racconti mitici ${ }^{38}$. La difesa dalle teorie primitiviste è condotta con una grande attenzione al divenire storico e alle specificità della civiltà romana a differenza di quanto accadeva nei suoi scritti precedenti, dallo studioso indicati

35 DuMÉzIL 1952, 81: Les uns sont surtout frappés par le fait de la structure, qui leur paraît commander, éclairer après les avoir triés, les éléments qui la composent. Pour eux l'ensemble ne se réduit pas à la somme des éléments ni ne peut s'expliquer par une accumulation fortuite, suivie d'accommodation, non plus qu'une chaise n'est simplemente la somme des morceaux de bois, des brins de paille, des clous de fer, qui ont servi à la fabriquer. Ils pensent donc que la grande affaire est de dégager la structure comme telle, avec ce qu'elle signifie, et que le problème ou plutôt les problèmes de l'origine précise des divers éléments se posent, certes mais sont moins importants, peuvent même à la rigueur, sans compromettre l'étude principale, rester sans solution.

36 DuMÉzIL 1992, 101-102.

37 DuMÉZIL 1992. 102-104.

38 DuMÉzIL 1952, 130-131. 
in calce al capitolo, in cui tali teorie venivano rifiutate in base ai consueti parallelismi con altri popoli parlanti lingue indoeuropee sullo sfondo dello schema trifunzionale.

\section{Conclusioni}

lla luce dei documenti inediti che ho preso in considerazione, il mutamento di orien-
tamento che si registra nella produzione di Dumézil a partire dai primi anni '50 e che
trova una sua prima realizzazione nell'ultimo capitolo del volume Les dieux des Indoeuropéens, può essere considerato, almeno parzialmente, frutto di un contatto diretto con la psicologia storica di Ignace Meyerson.

Il rapporto intellettuale con lo psicologo francese si colloca in una fase della produzione scientifica di Dumézil marcata dalla consapevolezza della necessità di rinsaldare le fondamenta teoriche su cui poggiavano i risultati empirici cui egli riteneva di essere giunto. In tale fase lo studioso mostra una certa apertura a influenze estranee al proprio profilo culturale, come il marxismo. L'indoeuropeista aveva preso coscienza del contrasto esistente tra le teorie sociologiche di matrice durkheimiana, di cui si avvaleva al fine di giustificare l'origine dell'ideologia trifunzionale, e la possibilità di ritrovare la medesima struttura operante in contesti storici differenti. L'influsso esercitato dalla psicologia storica contribuì a mitigare l'eccesso di "fissismo" proprio del trifunzionalismo, spingendo Dumézil a interrogarsi maggiormente sulle trasformazioni subite dall'ideologia indoeuropea nel corso dei secoli. Tale mutamento di prospettiva non risolveva realmente il nodo problematico che nelle lettere esaminate emerge in termini estremamente chiari. L'ideologia trifunzionale continuava a essere considerata una realtà fattuale ereditata da tutte le culture dei popoli parlanti lingue indeuropee. A tal proposito è esemplare l'introduzione a Mythe et épopée III, dove Dumézil individua nella presunta parentela genetica la giustificazione del ripresentarsi di elementi comuni nelle culture indoeuropee, elementi la cui origine si colloca nella sfera dell'ultra-storia ${ }^{39}$.

Bisognerà pertanto constatare come l'abbandono di una spiegazione puramente sociologica da parte di Dumézil non sia stata realmente sostituita con una teoria storica capace di rendere pienamente conto dell'operare dell'ideologia trifunzionale al di fuori del contesto che poteva averla originata.

Stefano Acerbo

Università di Pisa

Dipartimento di Filologia, Letteratura, Linguistica

Sezione di Filologia Classica - Greco

Via Galvani 1

56126 Pisa

acerboste@gmail.com 


\section{Appendice}

Vengono qui riprodotte le tre lettere inviate da Georges Dumézil a Ignace Meyerson esaminate nell'articolo.

I tre puntini di sospensione rinchiusi all'interno di parentesi quadre [...] indicano una singola parola di cui non mi è stato possibile fornire una lettura sicura. Al contrario sono dello stesso Dumézil i tre puntini di sospensione non all'interno della parentesi.

Le lettere contenute tra le due parentesi uncinate $<>$ sono mie integrazioni che sciolgono abbreviazioni puntate di nomi propri facilmente desumibili dal contenuto della lettera.

Infine i diversi tipi di sottolineatura che si ritrovano nella terza lettera riproducono, nella maniera più fedele possibile, i segni grafici utilizzati dallo stesso Dumézil.

\section{Testo 1}

G. Dumézil

82 rue N.D. des Champs

Paris $6^{\text {ème }}$

6 février 1949

Cher monsieur,

Je vous dois bien des remerciements pour votre beau livre pour les pages amicales aussi que vous avez consacrées à mon Loki dont j'ai lu les épreuves. Si j'étais plus jeune, j'essaierais de doubler d'analyses psychologiques l'exégèse un peu lourdement sociale que je fais des mythes. Mais l'âge est l'âge : je puis encore fonctionner dans le sens où je suis "remonté ", mais impossible de modifier le mécanisme.

Ma prière : un garçon (il a 32 ans) ${ }^{40}$ est mon étudiant depuis 1937 (interruption, pourtant, de 1941 à la fin de la guerre, car il a dû alors " disparaître ») et qui, justement s'intéresse passionnément aux mythes, et dans mon sens, et du point de vue psychologique, psychiatrique, souhaite s'accrocher, pour travailler ad aeternum à la Recherche Scientifique. C'est Lucien Gerschel $^{41}$, dont j'ai publié l'an dernier un petit mémoire à la fin de mon Jupiter, Mars, Quirinus $I V$ (tome LXII - et, provisoirement, dernier de la Bibliothèque de notre section des Hautes Études) je donne de lui à la RHR un joli article sur "Guerre et paix » dans les légendes de fondation des Saliens ; il a prêts un certain nombre de mémoires brefs sur des points de mythologie très divers, orientés vers vos études : sur le symbolisme des clefs ; sur de curieuses rencontres des techniques antiques de divination avec la psychanalyse, etc.

J'aimerais que vous entriez en relation avec lui pour que, si ce garçon très érudit et très sérieux vous paraît aussi utilisable qu'à moi-même, vous l'introduisez au CNRS, où je n'ai nulle accointance ni autorité. Je l'aurais aguillé vers l'enseignement si des disgrâces physiques ne l'y rendaient impropre.

Mais sa vraie voie est ailleurs.

40 Il testo tra parentesi è scritto nel margine sinistro del foglio.

41 Lucien Gerschel (1906 - 1985) fu prima studente e poi collaboratore di Dumézil: si occupò del persistere delliideologia trifunzionale nel folklore tedesco così come del carattere indoeuropeo di personaggi della storia antica di Roma quali Coriolano. 
Voulez-vous me dire si, à priori, la chose vous paraît possible ? Après (mais allez vite : il faudrait prendre une décision à temps pour la proposition du CNRS - le 15 mars, je crois).

Je lui dirais de vous expliquer son domaine, ses moyens et de vous envoyer quelques mémoires.

Si vous passez souvent à Paris, comme je crois, accepteriez-vous de le rencontrer ?

Dites-moi cela en toute simplicité.

Je vous en remercie et vous adresse mes plus sympathiques souvenirs.

Georges Dumézil

\section{Testo 2}

Paris le 24-4-49

Mon cher collègue,

Je vous suis infiniment reconnaissant du plaisir que me donnent vos articles. Je n'ai pas encore lu le "Journal" mais quelle lucidité, quelle humanité, quelle profondeur, dans le " chaudron "! Quant aux discontinuités, vous me trouvez conquis d'avance par mes expériences personnelles de chercheur et par les quelques coups d'œil que j'ai pu jeter sur le monde ; votre refus du "rationalisme simpliste" et de l'"empirisme naif" qui intéressent tant de discussions est libératoire, cette mise en évidence des rythmes, des crises, des risques, des métamorphoses, des mutations ; à côté des successions, des progrès, des développements évolutifs, rétablit l'équilibre systémique et philosophique de la recherche.

$\mathrm{Ci}$-joint le projet - repentir de Gershel. Je n'écris pas à P. M. Schuhl ${ }^{42}$, qui ne me connaît guère, je crains, et qui n'a, peut-être, pas le dossier. Servez-vous en séance, s'il y a lieu, du papier ci-joint : soit pour renfoncer un rapport favorable soit pour corriger une impression fâcheuse. Je crois seulement que si M. Piganiol ${ }^{43}$ est dans votre jury, une mention trop explicite de mon patronage ne déchaîne une vive opposition du dit : nos relations traversent une période difficile. De toute façon après la séance du 7 mai, aurez-vous la complaisance de me téléphoner ou de me dire, dans un billet, ce qu'on a fait de cette candidature.

Je tâcherai d'être libre le 13, mais ce ne serait pas avant 15 heures.

En toute sympathie et en remerciant de ces belles choses.

Georges Dumézil

\section{Testo 3}

Paris 18 novembre 1950

Mon cher collègue et ami,

Je vous dois de bien difficiles excuses, difficiles, car comment les agréerez-vous, actif toujours comme vous l'êtes, quand je vous dirai que le mal vient d'une soudaine impossibilité de rédiger, de juger, de décider, qui me stérilise depuis plus d'un an ? J'ai pu, [...] [...], préparer en détail mon cours de l'an dernier. J'aborde celui-ci sans même cette garantie ... Votre lettre, cet été, m’avait pourtant beaucoup touché, et avait avivé la honte de cette impuissance. Wikander étant venu me voir, je lui avais dit ce que vous paraissiez souhaiter - et n’avais pas remarqué

42 Pierre-Maxime Shuhl (1947-1972) fu storico della filosofia e in particolare del pensiero greco arcaico.

43 André Piganiol (1883-1968) fu uno storico e archeologo della civiltà romana. 
cette réserve, ou cette répugnance à faire symbiose avec une " psychologie » du type de la vôtre que vous me signaliez. Partant pour Amsterdam (où, actuellement, je ne suis pas allé), Wikander m'avait promis de vous écrire pour vous signaler, s'il y avait lieu, des communications ou interventions intéressantes. L'a-t-il fait ? Il m’a brièvement écrit, déçu semble-t-il (mais son mal à lui est plus profond, découragement, devant une situation temporale inextricable, depuis que Lund, puis Uppsala, lui ont préféré des médiocres). Ne m'accusez pas d'indifférence ou de lâcheté si je ne vous aide pas à composer ce numéro du "mythe ", si souhaitable. J'y écrierais bien volontiers, mais sans idées générales et sans perspectives : j'en suis devenu incapable. Et je ne " vois " pas l'ensemble, il y faut une habitude des hommes, une aptitude à harmoniser, qui sont deux des vos forces et que je n'ai pas du tout. Concevez votre plan. Si vous me faites l'amitié de me consulter sur un détail, il est possible que je me trouve un avis. Mais je ne domine pas. Puech ${ }^{44}$ sera lui plus qualifié que moi pour une délibération initiale.

En attendant j'ai achevé un petit papier qui vous intéressera peut-être (je pense qu'il va dans le sens de votre revue) où j'essaie de montrer comment, représentant l'histoire royale de Rome (dans De Republica) en fonction de préoccupations philosophiques, Cicéron qui fait subir des retouches exactement aux mêmes endroits (et dans le même sens) où la réforme zoroastrienne, dans ses préoccupations morales, a retouché la mythologie $\underline{\underline{m}}^{45}$ fonctionnelle des Indo-Iraniens. Comme je pense que cette mythologie - (ou théologie) indo - iranienne et cette histoire royale romaine sont bâties sur la même structure de pensée, le parallélisme des corrections qu'elles subissent l'une et l'autre, sont l'effet ici d'une philosophie politique, là d'une mystique moralisante, est un phénomène curieux, que je voudrais dégager, laissant aux autres le soin d'y réfléchir. Je serais curieux de voir l'explication marxiste de ces adaptations et rajeunissements de "superstructures" qui, ici et là, ne correspondent plus, depuis longtemps, à l'actualité sociale.

Gerschel m’a téléphoné qu'il avait vu monsieur Lévy-Bruhl ${ }^{46}$. Il lui a soumis deux ou trois projets juridiques dont il avait essayé de me parler ; mais je suis fermé à ces problèmes. Il semble (dixit $\mathrm{G}<$ erschel $>$ ) d'après la réaction de monsieur $\mathrm{L}<$ évy $>-\mathrm{B}<$ ruhl $>$, que cela a de l'intérêt. Tant mieux. Si G. accepte de se discipliner, dans une matière qui, évidement, l'intéresse, s'il reçoit les conseils de $\mathrm{m}<$ onsieur $>\mathrm{L}<$ évy $>-\mathrm{B}<$ ruhl $>$. (et bien entendu si monsieur $\mathrm{L}<$ évy $>-$ $\mathrm{B}<$ ruhl $>$ accepte aussi l'idée d'une recherche combinant d'une manière ou de l'autre ses idées et les miennes !), j'ai bon espoir qu'il se produira, tôt ou tard, des thèses plausibles. Je suis heureux de voir cet esprit agile, à la fois dévoué et défiant, accueillant et " autonomiste ", s’attacher à son nouveau maitre.

Nous verrons bien la suite...

J'espère que vous me ferez signe quand vous viendrez à Paris ? à la fin de novembre, nous saurions exactement le destin (la date du destin) de votre ami Henri Pieron ${ }^{47}$.

Je vous prie de recevoir, avec mes excuses, l'expression d'une fidèle sympathie.

Georges Dumézil

44 Henri-Charles Puech (1902-1986) fu uno studioso di storia delle religioni, particolarmente noto per i suoi studi sul manicheismo e lo gnosticismo.

45 È cancellata la parola < théologie $>$.

46 Henri Lévy-Bruhl (1884-1964), figlio di Lucien Lévy-Bruhl, fu un sociologo studioso del diritto romano in stretto contatto con Luis Gernet e Ignace Meyerson.

47 Henri Piéron (1881-1964) fu uno psicologo francese che pubblicò molti suoi studi sul Journal de psychologie normale et pathologique diretto da Ignace Meyerson. 


\section{Bibliografia}

Ampolo, Di Donato 1985

C. Ampolo, R. Di Donato, «Aspetti dell'opera di Georges Dumézil: una postilla», Opus 4 (1985), 217219.

Demoule 2014

J.-P. Demoule, Mais où sont passés les Indo-Européens? Le mythe d'origine de l'Occident, Paris 2014.

Di Donato 1990

R. Di Donato, Per una antropologia storica del mondo antico, Firenze 1990.

Di Donato 1999

R. Di Donato, Lingua e civiltà. Introduzione allo studio storico della lingua greca. Appunti e materiali, Pisa $1999^{2}$ (1996).

Di Donato 2013

R. Di Donato, Per una storia culturale dell'antico. Contributi a una antropologia storica, 2 voll., Pisa 2013.

DuCheSNE-Guillemin 2009

J. Duchesne-Guillemin, "Wikander, Oscar Stig», Encyclopedia Iranica, online edition, 2009, consultabile solamente online (http://www.iranicaonline.org/ articles/wikander-oscar-stig).

DumÉZIL 1948

G. Dumézil, Loki, Paris 1948.

DumÉZIL 1949a

G. Dumézil, L'héritage indo-européen à Rome, Introduction aux séries "Jupiter Mars Quirinus" et "Les mythes Romains", Paris 1949.

DumÉzIL 1949b

G. Dumézil, Le Troisième Souverain. Essai sur le dieu indo-arien Aryaman et sur la formation de l'histoire mythique de l'Irlande, Paris 1949.

DumÉZIL 1950

G. Dumézil, Chaire de Civilisation Indo-Européene. Leçon inaugurale faite le jeudi $1^{\text {er }}$ decémbre 1949. Collège de France, Paris 1950.

DuMÉZIL 1950b

G. Dumézil, «Les archanges de Zoroastre et les rois romains de Cicéron ", Journal de Psychologie Normale et Pathologique 43 (1950), 449-465.
DuMÉZIL 1952

G. Dumézil, Les dieux des Indo-européens, Paris 1952.

DumÉZIL 1973

G. Dumézil, Mythe et épopée III : histoires romanes, Paris 1973.

DumÉzIL 1992

G. Dumézil, Un banchetto di immortalità. Conversazioni con Didier Éribon, Parma 1992 [ed. or. Paris 1987].

ÉRIBON 1992

D. Éribon, Faut-il brûler Dumézil ? Mythologie, science et politique, Paris 1992.

García Quintela 2001

M. V. García Quintela, Dumézil. Une Introduction, Crozon 2001 [ed. or. Madrid 1999].

GinZBURg 1984

C. Ginzburg, «Mitologia germanica e nazismo: su un vecchio libro di Georges Dumézil», Quaderni di Storia 19 (1984), 857-882.

Meyerson 1948

I. Meyerson, Les Fonctions psychologiques et les cuvres, Paris 1948 [trad. it. a cura di R. Di Donato, Psicologia storica: le funzioni psicologiche e le opere, Pisa 1989].

Meyerson 1949

I. Meyerson, Compte rendu de Loki, G. Dumézil (Paris, 1948), Journal de Psychologie Normale et Pathologique (1949), 122-123.

Momigliano 1983

A. Momigliano, «Premesse per una discussione su Georges Dumezil», Opus 2 (1983), 329-341.

RIVIÈRE 1979

J.-C. Rivière, Georges Dumézil. À la déconverte des Indo-Européens, Paris 1979.

Timuş 2008

M. Timuş, «Quand l'Allemagne était leur Mecque: La science des religions chez Stig Wikander", in $\mathrm{H}$. Junginger (ed.), The Study of Religion under the Impact of Fascism, Leiden 2008, 205-222. 